\title{
Intracellular Metal Speciation in Streptococcus sanguinis Establishes SsaACB as Critical for Redox Maintenance
}

Cody J. Murgas ${ }^{\dagger}$, Shannon P. Green ${ }^{\ddagger \S}$, Ashley K. Forney ${ }^{\dagger}$, Rachel M. Korba ${ }^{\ddagger}$, Seon-Sook An ${ }^{\ddagger}$, Todd Kitten ${ }^{\ddagger \S *}$, Heather R. Lucas $^{\dagger *}$

† Department of Chemistry, Virginia Commonwealth University, Richmond, VA 23284

${ }^{\ddagger}$ Philips Institute for Oral Health Research, Virginia Commonwealth University, Richmond, VA 23298

$\S$ Department of Microbiology \& Immunology, Virginia Commonwealth University, Richmond, VA 23298

*corresponding authors: hrlucas@vcu.edu, tkitten@vcu.edu

\section{$\underline{\text { Table of Contents }}$}

Figure S1. Media Comparison

Figure S2. Intracellular Metal Content Comparison

Figure S3. Simulated EPR spectra for intracellular $\mathrm{Fe}^{3+}$ signal

Figure S4. Assays of Cellular Antioxidant Capacity

Figure S5. Comparison in iron-binding protein expression

Figure S6. Simulated EPR spectra for intracellular $\mathrm{Mn}^{2+}$ signal

Figure S7. NMR spectra indicating metal-bound DFO oxidation shift

Figure S8. UV/vis spectra indicating metal-bound DFO oxidation shift 


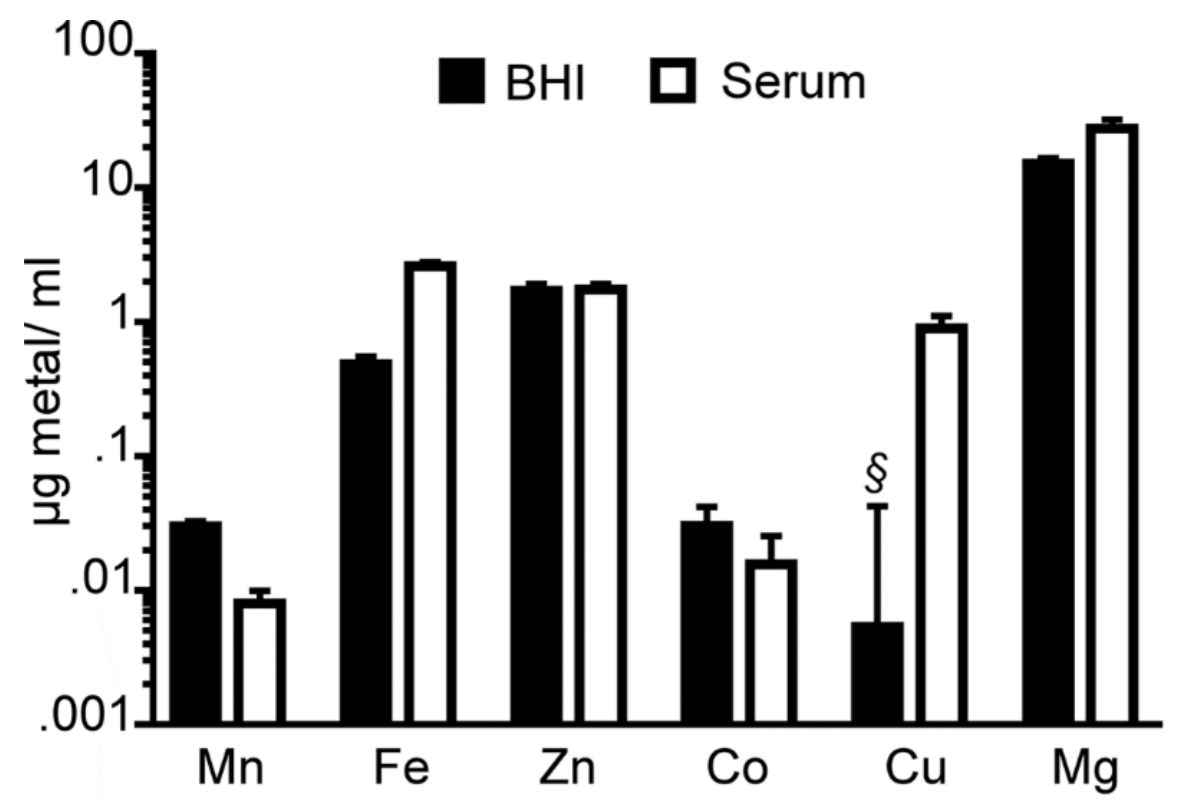

Figure S1. Media Comparison. Metal content in BHI media and pooled rabbit serum determined by ICPOES ( $\S$ - below the limit of detection) 
A
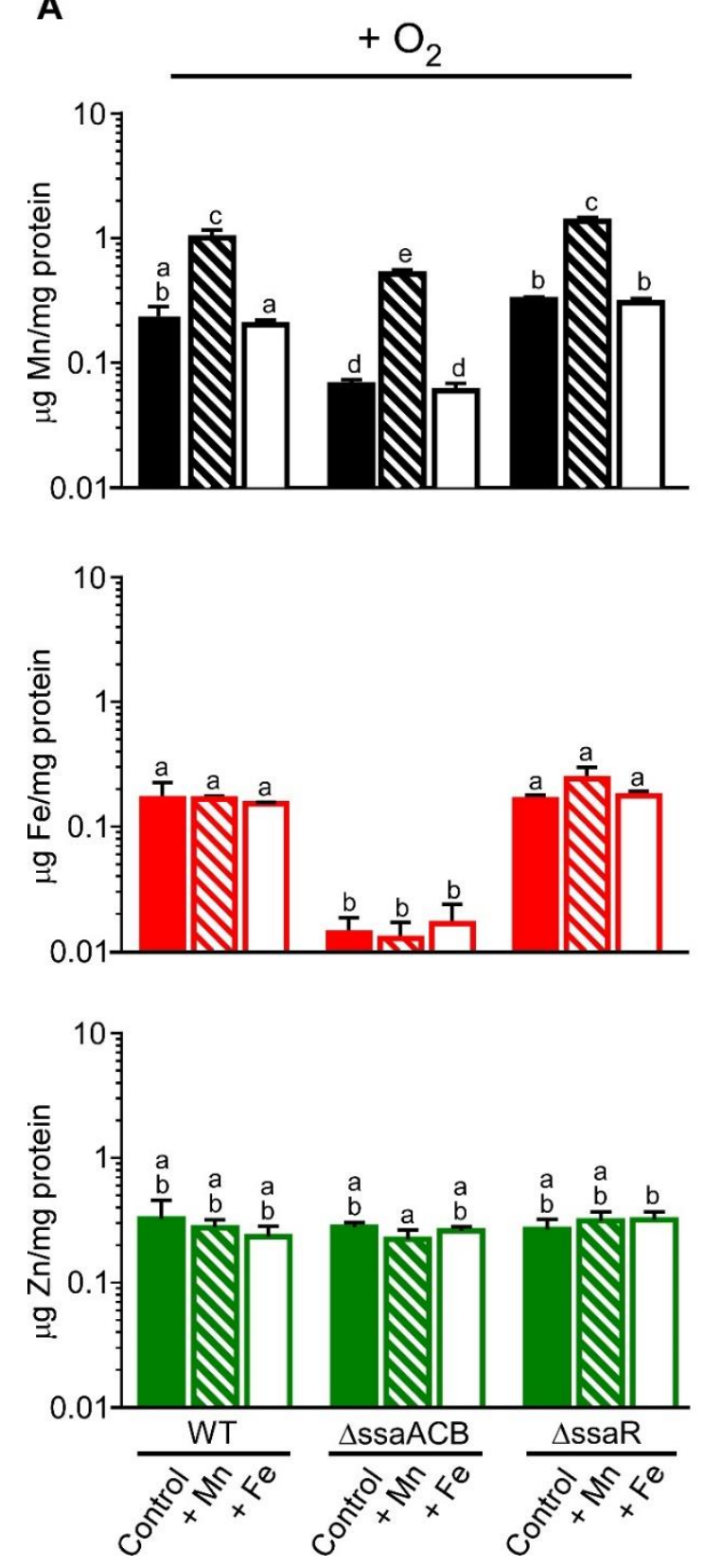

B
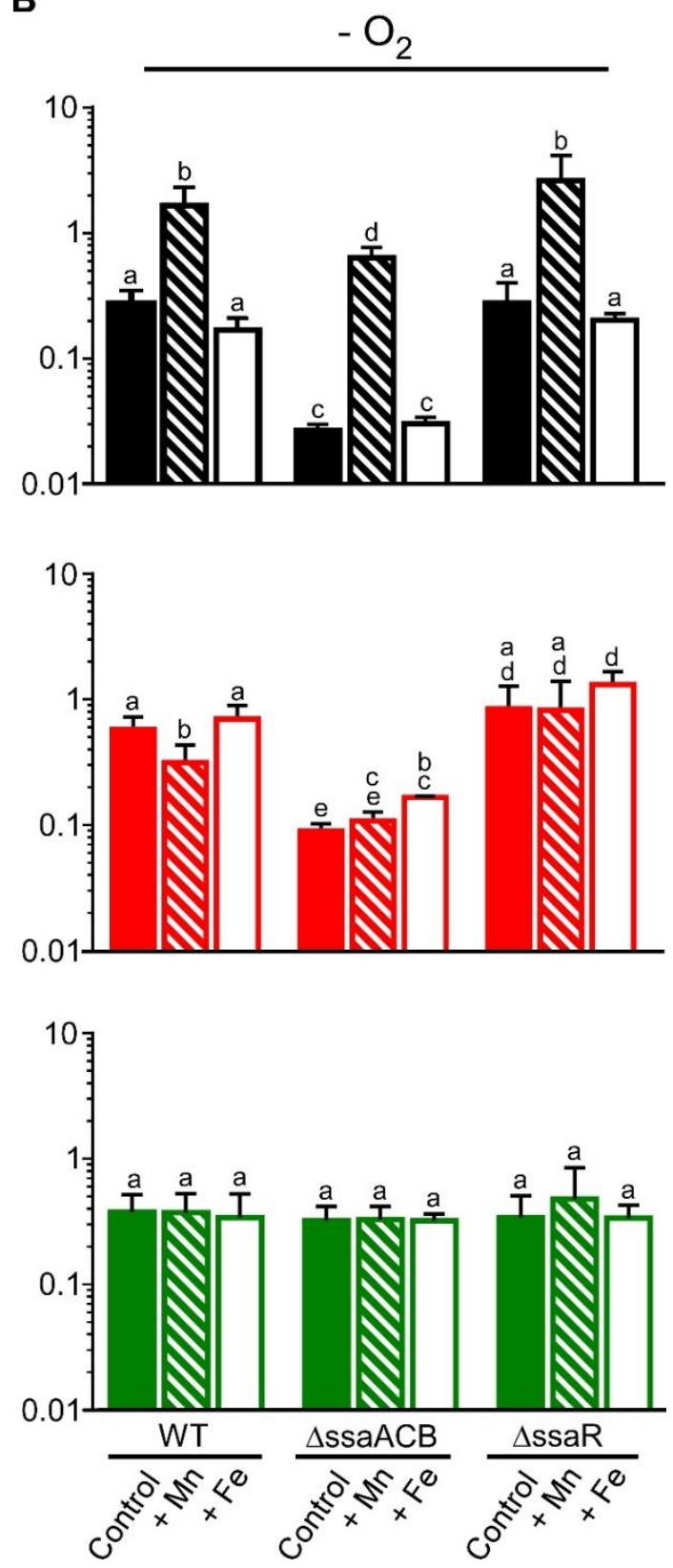

Figure S2. Intracellular Metal Content Comparison. The strains listed were cultured at $37^{\circ} \mathrm{C}$ in shaking flasks $\left(\mathrm{A},+\mathrm{O}_{2}\right)$ or an anaerobic chamber $\left(\mathrm{B},-\mathrm{O}_{2}\right)$ in $\mathrm{BHI}$ (Control) or $\mathrm{BHI}$ supplemented with the indicated metals at $10 \mu \mathrm{M}$. Cells were harvested by centrifugation and analyzed by ICP-OES as indicated in the Methods section. Means and standard deviations from 3 biological replicates are shown. Samples were compared by ANOVA or repeated-measures ANOVA, with a Tukey post-test performed when a significant difference was detected $(p<0.05)$. Bars that share a letter are not significantly different. Note that this experiment differs in $\mathrm{O}_{2}$ content from most of the others in this study and in the use of a log scale for the Y-axis. 

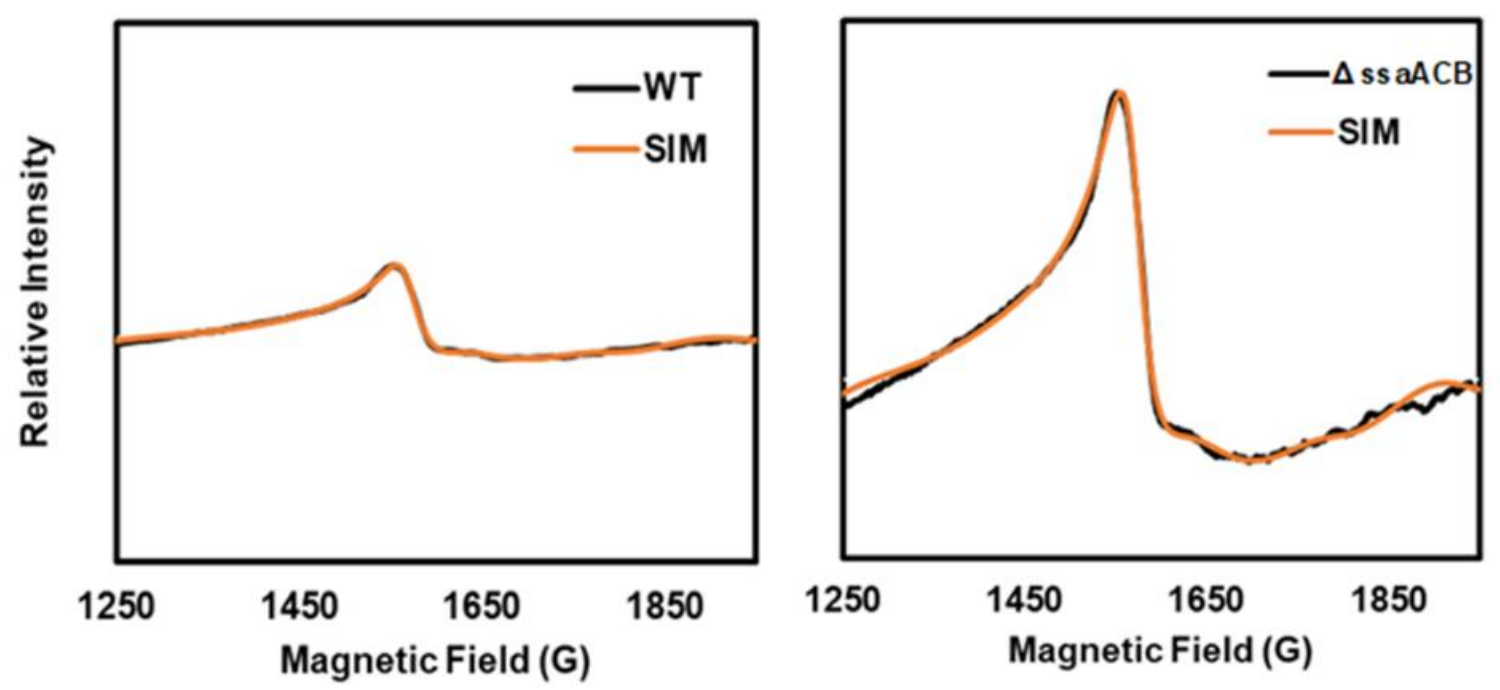

Figure S3. Simulated EPR spectra for intracellular $\mathrm{Fe}^{3+}$ signal. The experimental hyperfine coupling constant values were obtained by using the NIEHS WinSim program to simulate an approximate EPR spectrum, which was optimized by comparison with the experimental EPR spectrum (black) to produce the best simulated fit (orange). (Left) EPR spectra of the total $\mathrm{Fe}^{3+}$ content in WT observed and simulated; $v \approx 9.365511 \mathrm{GHz}$. (Right) EPR spectra of the total $\mathrm{Fe}^{3+}$ content in $\triangle$ ssaACB observed and simulated; $v \approx$ $9.366078 \mathrm{GHz}$. 
A

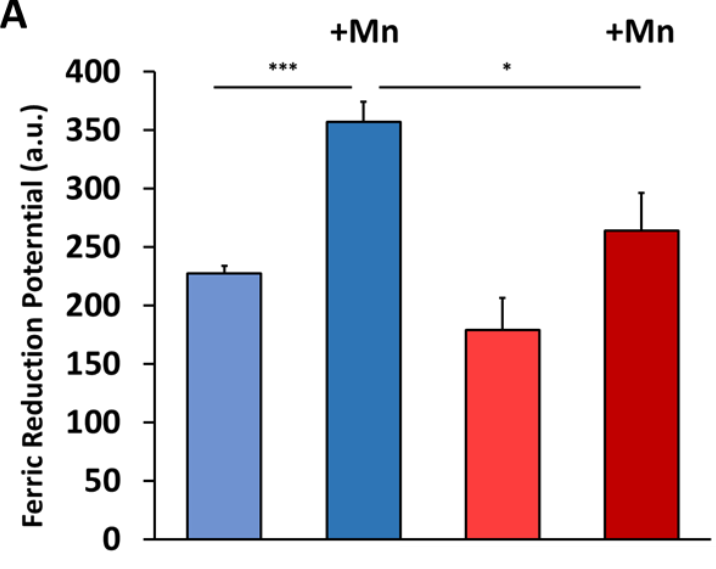

B WT WT $\quad \triangle$ ssaACB $\quad \triangle$ ssaACB

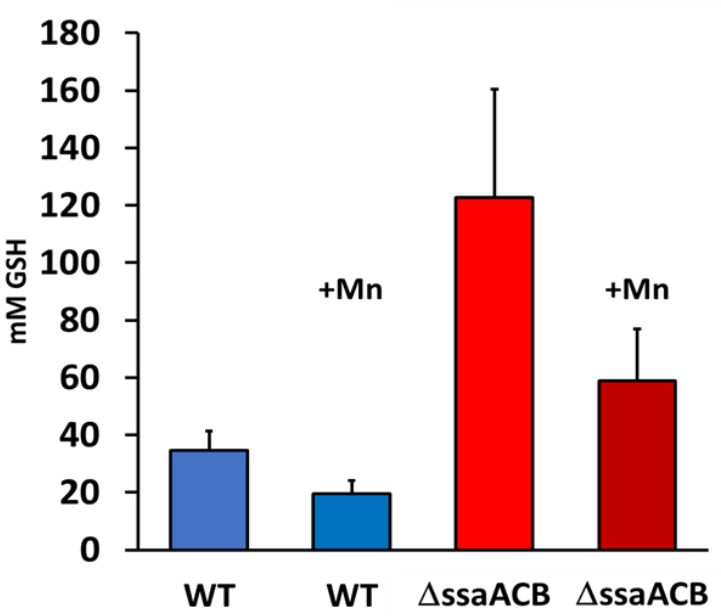

Figure S4. Assays of Cellular Antioxidant Capacity. (A) Ferric Reducing Antioxidant Power (FRAP) assay results measuring the collective capacity of enzymatic and nonenzymatic intracellular antioxidants for each strain grown in $\mathrm{BHI}$ media under Mn-supplemented and non-supplemented conditions. (B) Total intracellular glutathione levels measured for each strain grown in $\mathrm{BHI}$ media under $\mathrm{Mn}$-supplemented and non-supplemented conditions. 


\section{Changes In Fe-binding Protein Expression \\ Potassium Ferrocyanide Stain \\ Instant blue Coomassie}

"stain for iron binding proteins"

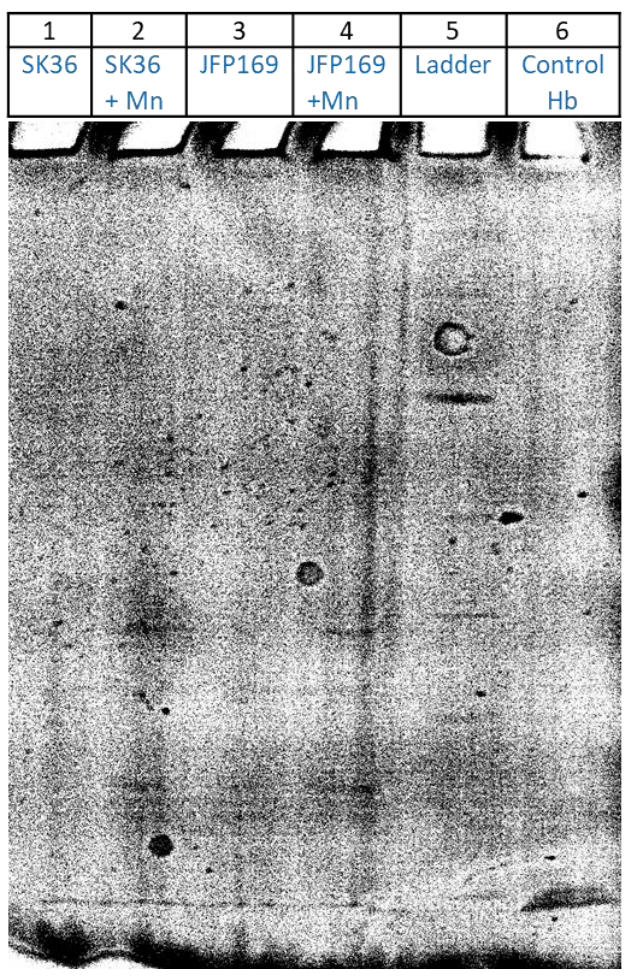

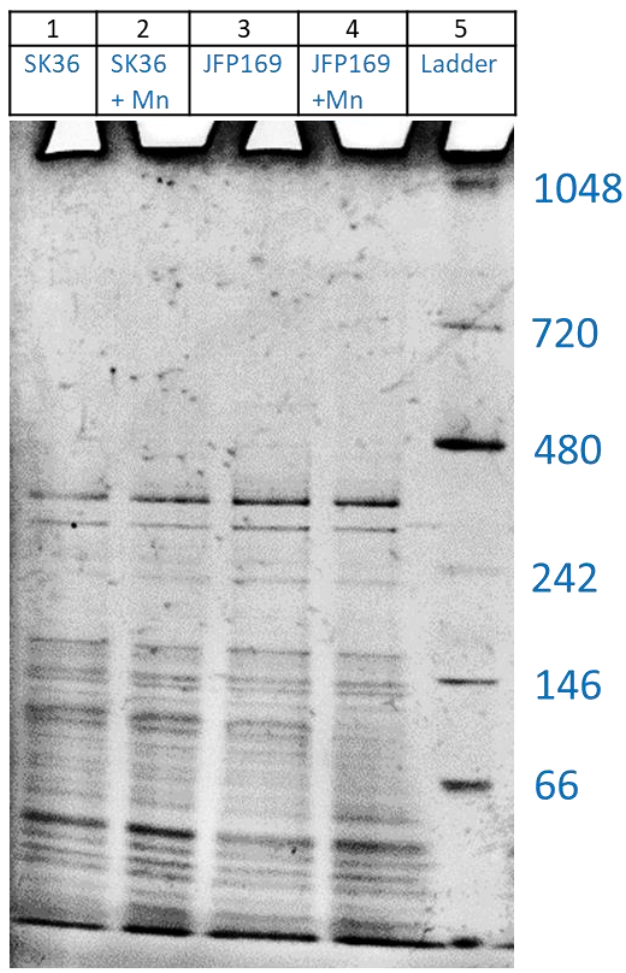

Figure S5. Comparison in iron-binding protein expression. Native gel stained with potassium ferrocyanide (left) and duplicate gel stained with Coomassie blue (right). Each lane is labeled according to the sample loaded: SK36 (WT), SK36 + Mn, JFP169 ( $\triangle$ ssaACB), JFP169+ Mn, and ladder. The native gel stained with potassium ferrocyanide is also loaded with a hemoglobin subunit as a control for the iron stain. 

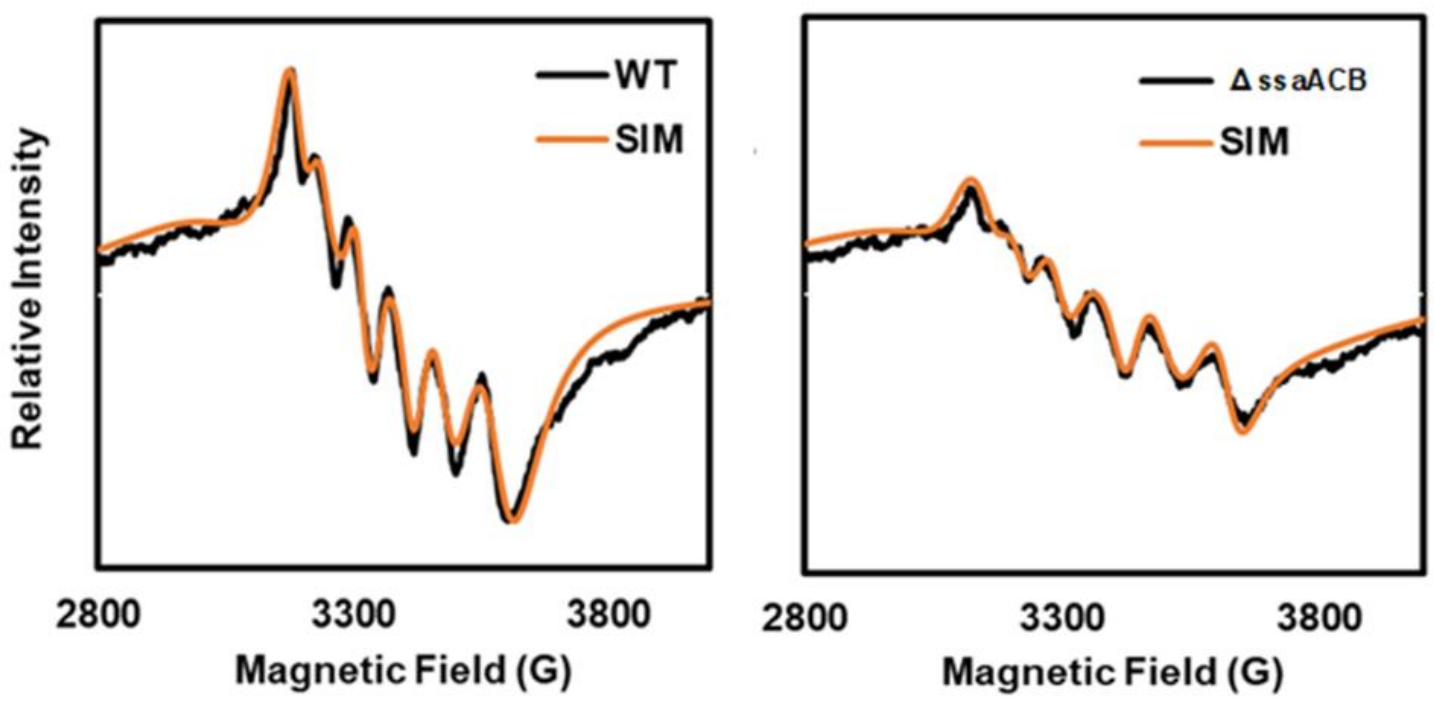

Figure S6. Simulated EPR spectra for intracellular $\mathrm{Mn}^{2+}$ signal. The experimental hyperfine coupling constant values were obtained by using the NIEHS WinSim program to simulate an approximate EPR spectrum, which was optimized by comparison with the experimental EPR spectrum (black) to produce the best simulated fit (orange). (Left) EPR spectra of the total $\mathrm{Mn}^{2+}$ content in WT observed and simulated; $\mathrm{v} \approx 9.375573 \mathrm{GHz}$. (Right) EPR spectra of the total $\mathrm{Mn}^{2+}$ content in $\triangle$ ssaACB observed and simulated; $\mathrm{v} \approx$ $9.377095 \mathrm{GHz}$.

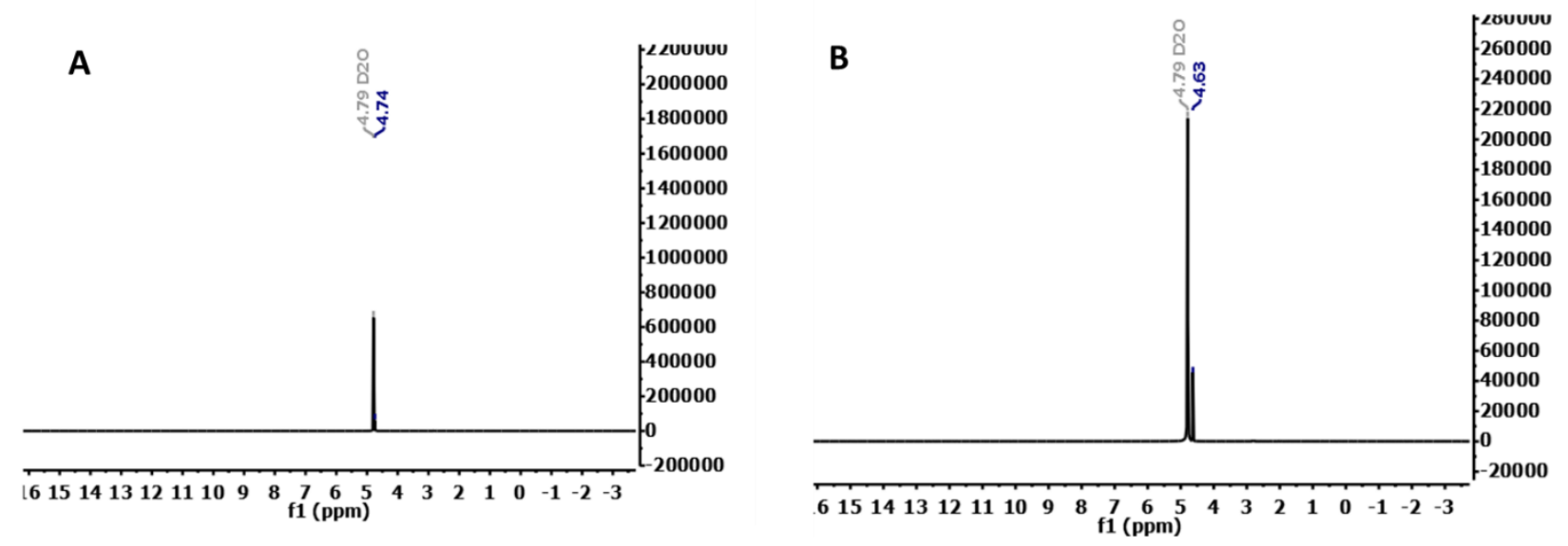

Figure S7. ${ }^{1} \mathrm{H}-\mathrm{NMR}$ of solvent peaks used to calculate the effective magnetic moments of $\mathrm{Mn}^{3+}$-DFO (A) and $\mathrm{Fe}^{3+}$-DFO (B) complexes under aerobic conditions $(\mathrm{n}=2)$. 

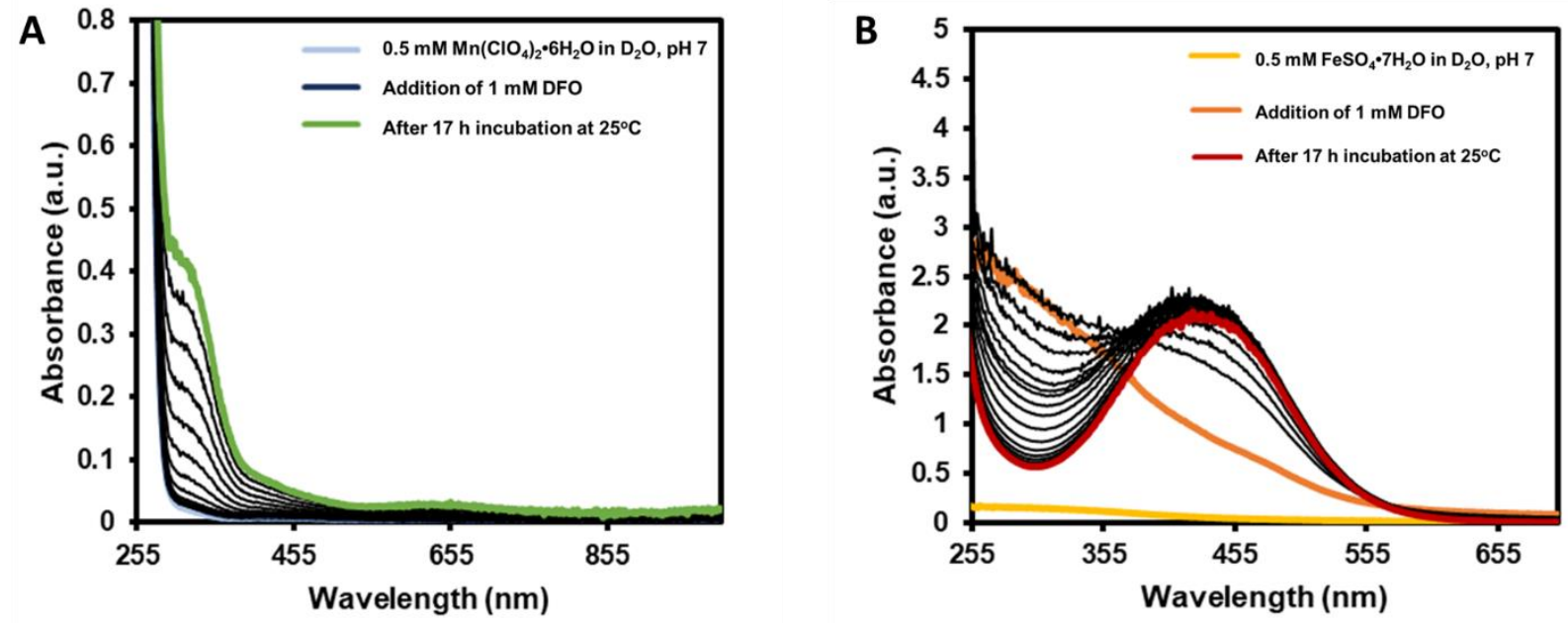

Figure S8. UV-Vis of $0.5 \mathrm{mM}$ solutions of A) $\mathrm{Mn}^{3+}$-DFO and B) $\mathrm{Fe}^{3+}$-DFO complexes compared to their respective starting metal salts in $\mathrm{D}_{2} \mathrm{O}(99.9 \%)$. 